\title{
Philosophiques
}

\section{Volker Peckhaus (Hrsg.) Oskar Becker und die Philosophie der Mathematik, München, Wilhelm Fink Verlag, 2005, 352 pages}

\section{Yvon Gauthier}

Volume 35, numéro 2, automne 2008

URI : https://id.erudit.org/iderudit/000446ar

DOI : https://doi.org/10.7202/000446ar

Aller au sommaire du numéro

Éditeur(s)

Société de philosophie du Québec

ISSN

0316-2923 (imprimé)

1492-1391 (numérique)

Découvrir la revue

Citer ce compte rendu

Gauthier, Y. (2008). Compte rendu de [Volker Peckhaus (Hrsg.) Oskar Becker und die Philosophie der Mathematik, München, Wilhelm Fink Verlag, 2005, 352 pages]. Philosophiques, 35(2), 612-613. https://doi.org/10.7202/000446ar d'utilisation que vous pouvez consulter en ligne.

https://apropos.erudit.org/fr/usagers/politique-dutilisation/ 


\section{$612 \cdot$ Philosophiques / Automne 2008}

(naturalisme et normativisme) ni aucune mention des différentes versions du mécanisme qui ont été développées depuis les vingt dernières années. En outre, pour ce qui est de l'ontologie des propriétés, on ne retrouvera aucun parallèle concernant les dispositions (propriétés = dispositions ?) et la façon dont nous pouvons les connaître.

Bref, Chasing Reality (2007) est un orphelin: 1) l'argumentation de Bunge dérape souvent, face aux sympathisants idéalistes et constructivistes, en accusations vindicatives (note 1 ) et ne constitue donc pas un dialogue «à tête reposée » chargé de faire avancer le débat; 2) ceux qui sont globalement matérialistes et mécanistes (systémistes ou non) ne trouveront rien de neuf dans cet ouvrage. En plus d'être essentiellement constitué des mêmes éléments que les livres précédents, surtout Emergence and Convergence (2003), Chasing Reality nous donne l'impression que Bunge a résolu, en principe, toutes les grandes questions philosophiques et qu'il nous laisse, au menu des tâches philosophiques futures, un vague mandat de philosophie "pratique » (morale et politique) (p. 281).

DAVY MOUGENOT

UQAM

\section{Volker Peckhaus (Hrsg.) Oskar Becker und die Philosophie der Mathematik, München, Wilhelm Fink Verlag, 2005, 352 pages.}

Oskar Becker (1889-1957) a été mathématicien avant de devenir un philosophe influencé, d'abord par Husserl, et ensuite par Heidegger (au grand dam de Husserl). Ses deux ouvrages principaux sont Mathematische Existenz (1927) et Grundlagen der Mathematik in geschichtlicher Entwicklung (1954), où il s'inspire de Schelling et de la philosophie de l'existence de Heidegger pour tenter de justifier l'existence d'ordinaux transfinis dans la théorie axiomatique des ensembles. Il a aussi contribué à l'histoire des mathématiques et à la logique modale, entre autres : ce qui a été appelé le postulat de Becker stipule que le statut des modalités est toujours régi par la nécessité, par exemple si A est possible, A est nécessairement possible. Ses prises de position politiques lui ont valu la désaffection du milieu intellectuel après la dernière guerre mondiale.

Le présent ouvrage, qui fait suite à une première parution consacrée aux rapports entre la philosophie et les sciences chez Oskar Becker (Die Philosophie und die Wissenschaften, 2002), est un recueil de quinze travaux publié sous la direction du réputé philosophe et historien de la logique, Volker Peckhaus. Peckhaus y va de deux contributions critiques dans ce volume, l'une consacrée à l'interprétation beckerienne de la notion formaliste d'existence chez Hilbert, et l'autre au rapport entre Becker et son collègue de Freiburg, le mathématicien Ernst Zermelo.

Peckhaus critique Becker à juste titre pour ne pas avoir pris la juste mesure du concept d'existence dans la métamathématique de Hilbert, une méprise philosophique qui est sans doute due à son parti pris réaliste. Pour appuyer sa thèse de la neutralité ontologique du concept d'existence chez Hilbert, Peckhaus cite la correspondance de Cantor et Hilbert, en 1897, sur l'existence de la totalité des alephs ou des cardinaux transfinis (taw ( $\eta$ ou encore la totalité des omégas ou ordinaux transfinis (notée $\Omega$ ). Cantor est conscient qu'il ne s'agit pas là d'un ensemble complété ou bien défini. 
Peckhaus nous dit que Cantor n'évoque pas le caractère antinomique de ces notions, mais Cantor décrète pourtant qu'il s'agit de pluralités absolument inconsistantes "absolut inkonsistente Vielheiten" (G. Cantor, Abhandlungen mathematischen und philosophischen Inhalts, E. Zermelo (dir.), Hildesheim, Georg Olms, 1966, p. 443). Même Russell, chasseur d'antinomies s'il en fut - peut-être à cause de son apprentissage hégélien - a dû reconnaître que Cantor l'avait précédé pour celle-là. L'auteur a sans doute voulu souligner le fait que Cantor n'avait pas une formulation logique du problème. C'est aussi l'essentiel de la critique qu'il adresse à Becker, qui a méconnu le sens formaliste de la notion d'existence dans sa version hilbertienne. Quant à Zermelo, il semble que Becker ait vu plus juste et que la construction cumulative des rangs ordinaux dans la hiérarchie transfinie des ensembles lui soit apparue quelque peu mystique et que sa consistance ou non contradiction ne soit plus qu'une question de goût ou une affaire de vision du monde (Weltanschauungsangelegenheit), selon une lettre de Becker à Zermelo du 31 décembre 1930 rapportée par Peckhaus (pp. 291-294).

Les autres contributions de l'ouvrage portent la marque des historiens, et la correspondance de Becker avec Heyting (M. van Atten), Hermann Weyl (P. Mancosu et T. Ryckman) ou Dietrich Mahnke (P. Mancosu) y est largement évoquée. Van Atten contribue aussi à un essai sur la réception phénoménologique (en particulier chez Becker) de la théorie brouwerienne des suites de choix. Des textes de E. Knobloch et de C. Thiel traitent de questions de mathématiques grecques dans le sillage des travaux de Becker en histoire des mathématiques. D'autres, A. Gigluano et H. Poser, sont plus résolument philosophiques et s'intéressent à la philosophie de l'existence ou à l'ontologie de Becker. Un article intéressant de J. Emrich note l'usage de la notion husserlienne d'horizon. Mais alors que l'horizon ouvert (der offene Horizont) permet à Becker de justifier l'existence des ensembles transfinis, ce que j'ai appelé ailleurs l'horizon constructif n'autorise que l'infini potentiel des suites infiniment processives ou $<$ effinies $>$ de l'intuitionnisme brouwerien (voir mes Fondements des mathématiques, Montréal, PUM, 1976, p. 106). Les deux contributions finales sur un fondement protothéorique inspiré par Becker et Lorenzen de la théorie des ensembles classique (P. StekelerWeithofer) et sur la triade Becker-Gentzen-Lorenzen (M. Wille) sont plus spéculatives et reposent sur des hypothèses philosophiques ou des bases historiques fragiles.

Au total, ce n'est pas toute la production scientifique de Becker qui est prise en compte dans cet excellent recueil. Les travaux de logique modale ne sont pas discutés dans le contexte de la philosophie des mathématiques, et les contributions revêtent rarement un caractère critique - à part les contributions de l'éditeur du recueil, comme je l'ai noté plus haut. On ne sait trop si la publication des actes de colloques, organisés par Anne-Marie Gethmann-Siefert et qui ont donné naissance à cet ouvrage, a pour mission de réhabiliter la figure controversée de Becker, mais il est certain que celuici a produit une œuvre philosophique assez importante pour qu'on ne puisse la soustraire à l'attention des historiens et à la vigilance critique. 